\title{
The Ages of High-Mass X-ray Binaries in M33
}

\author{
Kristen Garofali and Benjamin F. Williams \\ University of Washington Astronomy Department, Box 351580, Seattle, WA, 98195 \\ email: garofali@uw.edu, ben@astro.washington.edu
}

\begin{abstract}
We present initial results on a survey of high-mass X-ray binaries (HMXBs) in the nearby, star-forming spiral galaxy M33. The HMXB population in M33 is identified and characterized using a combination of deep Chandra X-ray imaging and archival Hubble Space Telescope (HST) observations. We determine ages for the HMXBs to $\sim 5 \mathrm{Myr}$ precision from fits to the color-magnitude diagrams (CMDs) of the surrounding stars and the resultant star formation histories (SFHs). The HMXBs in our M33 sample have measured ages, as well as candidate optical counterparts identified from HST photometry.
\end{abstract}

Keywords. X-rays: binaries, stars: evolution, surveys

We demonstrate a technique for identifying HMXB candidates and their potential optical counterparts using a combination of deep Chandra imaging and archival HST data. Ages for each candidate are determined from fitting the CMD of the stars surrounding the HMXB candidate. An example of this technique for a very young (10-20 Myr) candidate HMXB in M33 is shown in the Figure 1.

Previous studies of HMXB populations in the Small Magellenic Cloud, NGC300, and NGC2403 (Antoniou et al. 2010; Williams et al. 2013) have revealed HMXBs to have a preferred age of $\sim 30-60 \mathrm{Myr}$, implying large populations HXMBs containing Be donor stars. This survey in M33 will consist of a population of well-characterized HMXBs with measured ages in a large, star-forming spiral galaxy, and will place past HMXB studies in broader context by examining the interplay between host galaxy metallicity and SFH and their combined effects on massive star evolution in binaries.
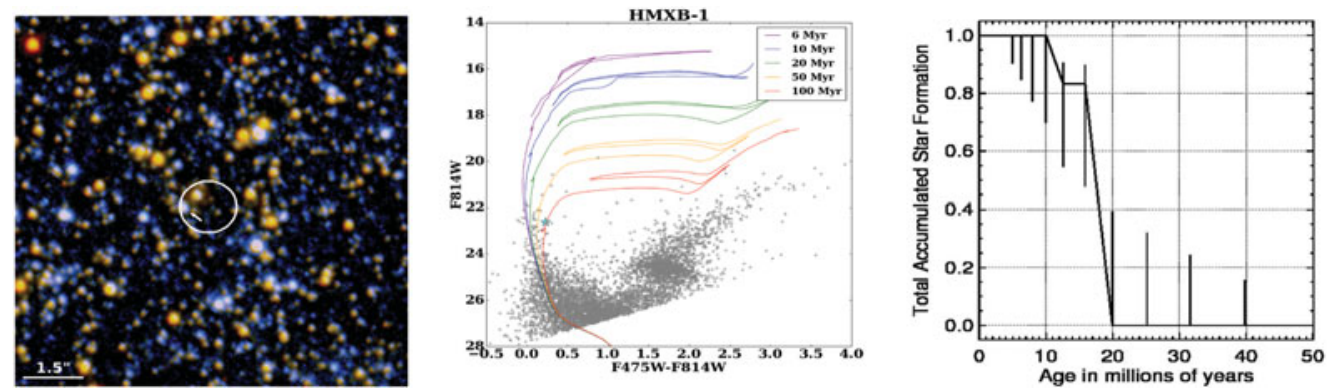

Figure 1. Left: The X-ray error circle $\left(0.7^{\prime \prime}\right)$ in white overlaid on a color HST/ACS image of a region in the disk of M33. The blue star, denoted by a white arrow, makes this a candidate HMXB. Center: The HST CMD of the stars within 50 pc of the HXMB candidate (cyan star). Padova group isochrones are overlaid for reference. Right: The cumulative age distribution based on CMD fitting for stars within 100 pc of the HMXB candidate.

\section{References}

Antoniou, V., Zezas, A., Hatzidimitriou, D., \& Kalogera, V. 2010, ApJL, 716, 140

Williams, B. F., Binder, B. A., Dalcanton, J. J., et al. 2013, ApJ, 772, 12 